\title{
Formation initiale des Enseignants de Physique-Chimie : l'Ouverture aux Nanosciences \& Nanotechnologies
}

\section{F. Dubreuil'1, A. Baudrant'2, Ch. Rambaud ${ }^{3}$, F. Marchi' 4,5}

1 Enseignant-chercheur à l'UFR de Chimie et biologie de l'Université Joseph Fourier, Grenoble 1

2 Ingénieur au CEA-LETI, Grenoble

3 Enseignant-chercheur et co-responsable du Master ME2D (Master Enseignement second degré) de Physique-Chimie à l'UFR PHITEM de l'Université Joseph Fourier, Grenoble 1

${ }^{4}$ CIME Nanotech, 3 parvis Louis Néel, BP 257, 38016 Grenoble cedex 1

${ }^{5}$ Enseignante-Chercheuse à l'UFR de PHITEM de l'UJF et responsable de l'UE «Objets quotidiens, Nanosciences et Education» du master ME2D de Physique-Chimie.

\section{Résumé:}

En 2010 la refonte de la formation des enseignants du primaire et secondaire a conduit à la création des Master Enseignement ; c'est dans ce cadre que l'Université Joseph Fourier a décidé de consacrer une unité d'enseignement (UE) dite d'ouverture à la recherche aux « Nano » pour les futurs professeurs de physique et de chimie. Cette UE intitulée "Objets quotidiens, Nanosciences et Education" unique en France a la particularité de s'appuyer sur des plateformes d'enseignement expérimental de haut niveau dans ce domaine. De plus elle propose une évaluation des connaissances innovante qui repose sur la conception d'une séquence d'enseignement sur une thématique «Nano» dédiée à des élèves de niveau première ou Terminale scientifique.

Mots clés: Nanosciences, Nanotechnologies, Formation initiale des enseignants, Travaux pratiques, enseignement des sciences 'chaudes', microscopie en champ proche, Microscopie électronique, nanoparticule, cellule photovoltaïque, salle blanche

\section{Introduction}

En 2010, la réforme de la formation des enseignants a abouti à la «mastérisation » portant ainsi le niveau de qualification des futurs enseignants du primaire et du secondaire à Bac +5 .

A l'Université Joseph Fourier (UJF), cette réforme a donc conduit à la création de masters enseignement dont celui de Physique-Chimie (PC) destiné à former les futurs professeurs de collèges et lycées qui seront chargés d'enseigner ces deux champs disciplinaires [1]. Au sein de ce nouveau Master cohabite des unités d'enseignement (UE) de consolidation des connaissances en PC de base (niveau Licence), d'ouverture à la recherche, de pédagogie et mise en situation professionnelle. Le contenu des UE disciplinaires est défini par le programme du concours et ceux des UE de pédagogie et mise en situation sont définis par l'IUFM (Institut Universitaire de Formation des Maîtres, qui vont s'intégrer dans les futures ESPE -Ecole Supérieure du Professorat et de l'Education à la rentrée 2013) en lien étroit avec l'éducation nationale, celui de l'UE d'ouverture à la recherche restait à concevoir. Le souhait des responsables du master Enseignement $\mathrm{PC}$ fut donc de proposer une UE répondant à trois critères :

- Premièrement une UE qui sensibilise de manière approfondie les étudiants à une thématique de physique et/ou de chimie émergeante ou encore appelé «sciences chaudes » sur laquelle la recherche académique grenobloise soit en pointe,

- Deuxièmement une UE qui propose un mode d'évaluation original des connaissances et des savoir-faire acquis au cours de l'UE qui soit en lien direct avec des compétences professionnelles propres au métier d'enseignants à savoir la conception d'une séance d'enseignement,

- Troisièmement une UE qui puisse ouvrir les portes d'une éventuelle poursuite et donc de réorientation dans un master 2 Pro différent du master 2 enseignement. 
Depuis plusieurs années, les laboratoires Grenoblois sont en pointe dans le domaine des Nanosciences et des micro et nanotechnologies et les universités disposent d'un centre d'enseignement et de formation qui leur est dédié à savoir le CIME-Nanotech [2]. D'autre part depuis la création en 2006 du master $\mathrm{N}^{2}$ "Nanosciences\&Nanotechnologies" [3] qui comprend trois mentions "recherche" et une mention Professionnalisante, l'UJF propose une formation de qualité dans ce domaine dont la mention Pro est en parfaite adéquation avec le bassin d'emploi grenoblois. De plus la physique et la chimie jouent un rôle clé dans plusieurs domaines d'application des Nanosciences; par exemple dans les procédés de fabrication et de caractérisation des micro et nano-composants qui sont au cœur du codage de l'information sur lequel repose la société du numérique qui révolutionne nos modes de vie ou encore dans l'élaboration de nouveaux matériaux à base de nanoparticules qui seront au coeur des futures technologies de stockage de l'énergie. Enfin, au cours des années 2007-2010, un petit nombre (3 cas) de titulaires du CAPES ou de l'agrégation de physique-chimie qui pour la plupart ont exercé le métier d'enseignant quelques années, ont postulé et intégré le Master 2Pro IMN (Ingénierie des Micro et Nanostructures) dans le but de changer de carrière professionnelle. Tous ont obtenu leur Master 2 Pro IMN de manière fort honorable (mention très bien ou Bien) et surtout ont réussi sans soucis particulier à intégrer le monde de l'entreprise et à obtenir un CDI ou CDD à l'issue de leur stage comme la plupart des étudiants de ce Master Pro.

A partir de cette analyse du paysage de la recherche, du bassin industriel de cette région, de l'offre de formation ainsi que de l'impact des nanotechnologies dans notre société, il est apparu pertinent de sensibiliser ces futurs enseignants aux Nanosciences et Nanotechnologies.

Ainsi, cette UE d'ouverture a été intitulée : “Objets du quotidien, Nanosciences et Education”. Elle s'inscrit dans une double approche : (1) Faire-acquérir des bases théoriques et expérimentales en nanophysique et en nano(bio)chimie grâce notamment à de nombreux Travaux Pratiques (TP) ; (2) un contrôle des connaissances original basé sur l'élaboration d'une séance de classe de niveau 1ère ou Terminale S portant sur un thème «Nano».

C'est dans cet esprit que depuis maintenant trois ans est dispensée cette UE de 48h qui se compose de $28 \mathrm{~h}$ de TP, $16 \mathrm{~h}$ de cours et $4 \mathrm{~h}$ d'exposé. Le cours et les TP sont assurés par deux enseignants-chercheurs et un ingénieur du CEA. Les TP sont dispensés au CIME-Nanotech [2] et sur le plateau de Chimie de l'UJF [4]; leur contenu est décrit dans la section 2. Le cours se décline en trois parties: nanophysique $(7 \mathrm{~h})$, nanochimie $(7 \mathrm{~h})$ et introduction à la micro/nanoélectronique (2h). Enfin le contrôle des connaissances est basé sur la réalisation d'un dossier puis d'un exposé sur un sujet "Nano" et de son adaptation à une classe de lycéens. Le contenu et les modalités de ce travail sont détaillés dans la section 3. Ce travail est évalué par un jury composé des enseignants-chercheurs responsables de l'UE, du responsable du master et un ou deux représentant(s) de l'éducation nationale (inspecteur et/ou enseignant du secondaire) impliqué dans les actions de l'académie grenobloise menées dans le cadre du programme «Nano@school» [5], déclinaison locale du projet national Nanoécole [6]. A ce jour la déclinaison grenobloise de cette action nationale, se traduit par l'accueil au CIME-Nanotech d'une douzaine de classe de première $S$ par an (une journée par classe).

Comme indiqué sur le site web de Nanoécole [6], l'objectif de ces actions se résume ainsi : «le projet national Nanoécole vise à expérimenter l'introduction des nanotechnologies à l'école. L'idée principale consiste à associer enseignement scientifique et réflexion socio-scientifique. Nous oeuvrons pour un enseignement scientifique visant à donner aux citoyens de demain les clés pour pouvoir participer aux choix technologiques à venir et renforcer le dialogue entre science et société.»

Pour atteindre ce type d'objectif, une sensibilisation des enseignants de sciences à ces thématiques émergeantes à fort impact sociétal est indispensable. Le présent article est dédié à l'UE d'ouverture «Objets du quotidien, Nanosciences et Education» dispensée aux enseignants en formation initiale au sein du master second degré de physique-chimie de l'université Joseph Fourier. La dernière section de l'article est consacrée au retour d'expérience et aux perspectives. 


\section{Immersion Expérimentale au cœur des Nano}

Une très large place est réservée aux Travaux Pratiques pour donner un sens concret aux notions théoriques abordées dans le cours ainsi que pour permettre aux étudiants de se familiariser avec les technologies et les environnements de travail très spécifiques aux nanosciences et nanotechnologies. Pour cela, les séances de TP se déroulent au sein du CIMENanotech notamment en salle blanche et sur la plateforme Nanomonde [7] ainsi que sur la plateforme de chimie analytique -PCA- [4] de l'UJF. La PCA récemment créé, s'appuie sur un réseau de laboratoires (CERMAV, etc.) qui ont développés depuis plusieurs années des compétences et savoir-faire en nanochimie.

Les Travaux pratiques comportent trois volets.

-Le premier est dédié à la Micro/Nano-fabrication (8h) en salle banche où les étudiants se sensibilisent à cet environnement de travail particulier en fabriquant une capacité MOS ;

-Le deuxième est dédié à l'observation, la mesure et la manipulation à l'échelle nanoscopique d'objets et/ou de phénomènes quantiques (16h) dont trois séances de $4 \mathrm{~h}$ se déroulent sur la plateforme Nanomonde [7] et une de $4 \mathrm{~h}$ dédiée à la microscopie électronique est effectuée au laboratoire du CERMAVi.

Les techniques de microscopies en champ proche sont utilisées pour étudier l'effet tunnel (phénomène lié à la mécanique quantique) et les forces prédominantes à l'échelle nanoscopique (Van der Waals, capillarité, etc.). Grâce à ces techniques "l'intimité physique et chimique» des objets quotidiens emblématiques tels que des surfaces de disques optiques ou encore d'une couche de graphite qui compose la mine d'un crayon à papier est observée et analysée.

Un «serious game», le NanoLearner [8,9] permet de s'immerger à travers les sens humains, dans le nanomonde et d'appréhender la notion compliquée de contact à l'échelle nanoscopique et ses implications sur la manipulation d'objets submicroniques.

Au cours de la séance de $4 \mathrm{~h}$ de MEB/TEM la structure d'un polymère alimentaire l'amidon, est étudiée.

-Le troisième volet aborde les "Nano" pour l'énergie en $4 \mathrm{~h}$ à travers la réalisation et la caractérisation d'une cellule photovoltaïque à colorant de type Graetzel qui nécessite l'élaboration de nanoparticules, ce TP se déroule sur la plateforme de chimie analytique de l'UJF.

\subsection{Nanofabrication}

Au cours de ce TP de 8h en salle blanche du CIME-Nanotech, les étudiants réalisent une capacité MOS sous la responsabilité d'un ingénieur du CEA-LETI. Cette séance est précédée d'un cours introductif à la micro/nano-électronique où sont notamment abordées les techniques de micro et nano-fabrication et leur évolution ainsi que les enjeux de la miniaturisation des composants (la fameuse «loi » de Moore).

Ce TP s'appuie sur un polycopié standard qui sert de base à l'ensemble des formations de niveau L3 à master qui désirent offrir un TP d'initiation à la micro/nano-électronique. Au cours de cette séance, les étudiants réalisent les étapes de fabrication du composant sur silicium: nettoyage et désoxydation par HF (acide fluorhydrique) avant oxydation par traitement thermique (figure 1), lithographie optique avec et sans alignement, gravure humide ou sèche du $\mathrm{SiO}_{2}$, dépôt métallique par pulvérisation cathodique. A la fin de la séance, une visite de toute la salle blanche remémore les procédés utilisés pour la fabrication de circuits complexes et situe le TP par rapport à l'état de l'art en production de circuits avancés [10].

Afin d'adapter ce TP aux préoccupations et intérêt plus large de la formation initiale des enseignants, le CR ne se focalise pas uniquement sur les aspects technologiques mais s'ouvre sur un questionnement diversifié : (1) les échelles de grandeurs du composant fabriqué et de ses parties actives par rapport à la taille d'objets naturels (cheveux, cellules, virus, etc.); (2) les sources de contaminations possibles qui sont communes à tous les environnements salle

i CERMAV: Centre de Recherche sur les macromolécules végétales, http://www.cermav.cnrs.fr/ 
blanche et salle propre telles que les salles de préparation en agro-alimentaire ou encore au bloc-opératoire et comment les minimiser. (3) les différents métiers et niveaux de compétences nécessaires au maintien et à l'amélioration du savoir faire d'une telle industrie (physique, métallurgie, chimie, informatique,....mais aussi traitement et distribution de l'air, de l'eau, des gaz et des effluents....et encore maintenance des équipements, des bâtiments ....)

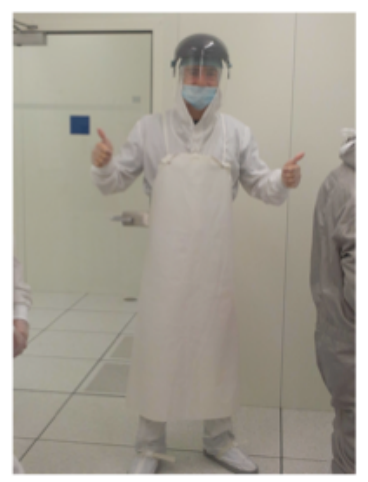

Figure 1: étudiant en tenu de protection pour l'utilisation des bains de HF lors de l'étape de désoxydation du Si

\subsection{Caractérisation des surfaces par microscopie en champ proche}

Les microscopies en champ proche telles que la Microscopie à Force Atomique (AFM) et la Microscopie à effet tunnel (STM) sont des techniques phares (prix Nobel de physique en 1986 décerné à Gerd Binnig et Heinrich Rohrer pour la conception du premier STM) pour l'étude et la caractérisation des surfaces et objets de l'échelle micronique à atomique.

Dans le cadre de cette UE d'ouverture, nous avons décidé d'orienter ces deux séances de $4 \mathrm{~h}$ chacune sur deux thématiques : (1) Etude par AFM du codage 'physique' de l'information à la surface d'un matériaux grâce à l'étude de CD, DVD puis Blu-ray; (2) l'étude par STM d'une surface de graphite HOPG (Highly ordered pyrolytic graphite) qui est une des formes allotropiques du carbone dont la structure cristalline et les propriétés électriques sont proches de deux formes emblématiques du carbone à l'échelle nano à savoir les fullerènes (prix Nobel de Chimie en 1996 décerné conjointement à Robert F. Curl Jr., Sir Harold W. Kroto et Richard E. Smalley) et le graphène (prix Nobel de physique en 2010 décerné conjointement à Andre Geim et Konstantin Novoselov).

\subsubsection{Etude de l'enregistrement de l'information sur disque optique par microscopies optique et à force atomique (AFM)}

Au cours de la séance d'étude des CD/DVD et Blue-ray, les étudiants apprennent à préparer ces surfaces pour les caractériser à l'AFM. Ensuite ils débutent leurs observations par le microscope optique équipée d'une caméra (microscope LEICA, modèle Z16 APO A), outil indispensable aux scientifiques, afin d'obtenir une première caractérisation des motifs de codage lorsque ces derniers présentent encore des dimensions accessibles à cet instrument (figure 2). Le passage du CD au DVD permet de s'approcher des limites de résolution d'un microscope optique puis le passage du DVD au Blue-ray permet de franchir cette limite et donc de questionner les étudiants sur l'origine de la résolution d'un microscope optique (critère de Rayleigh, défaut des optiques, etc.).
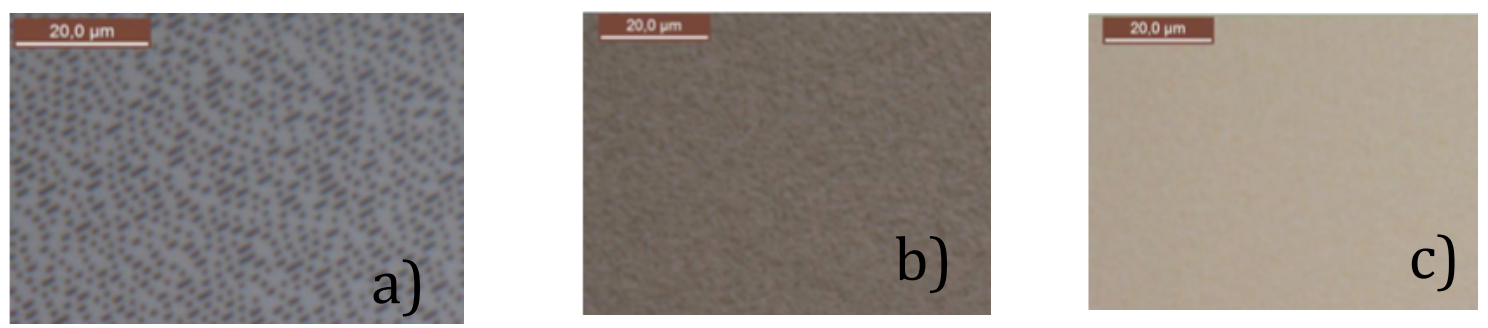

Figure 2: images de surface de CD(a), de DVD (b) puis de Blue-ray (c) obtenues par les étudiants en TP grâce aux microscopes optiques LEICA 
Le fait que les motifs du Blu-ray ne soient pas visibles au microscope optique introduit 'naturellement' le recours à d'autre type de microscopies telles que la microscopie à force atomique ou la microscopie électronique à balayage. Les notions liées à la dualité ondecorpuscule sont ainsi abordées à la fois lors de la séance d'AFM et celle de MEB. D'un point de vue instrumental, les étudiants règlent les paramètres de l'AFM notamment les gains de la boucle de contre-réaction, la vitesse de balayage et le point de régulation afin d'obtenir des images stables et reproductibles (Figure 3).

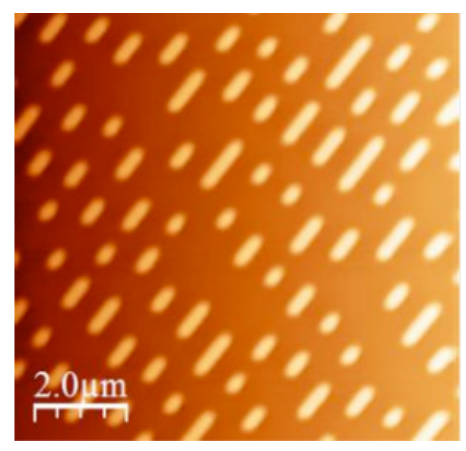

Figure 3 : Image AFM d'une surface de DVD obtenue en mode contact par les étudiants en TP, l'échelle de couleur code la hauteur de 0 à 300nm (rouge foncé à blanc).

\subsubsection{Etude d'une surface de graphite par microscopie à effet tunnel}

Cette séance débute par la préparation de l'échantillon, à savoir le clivage à l'aide d'un morceau d'adhésif de la surface du graphite HOPG, méthode connue sous le nom d'exfoliation mécanique. Ensuite ils réalisent des images de l'échelle submicronique qui leur permettent d'observer des marches de hauteur mono ou pluri-atomiques (figure 4a) puis ils réduisent la zone de balayage afin de visualiser l'arrangement atomique de la couche de graphite (figure $4 \mathrm{~b}$ ).

Le contenu et le polycopié du TP s'appuient sur celui déjà bien rodé proposé aux étudiants de master $\mathrm{N}^{2}$ de l'UJF [7]. Grâce aux images atomiques les étudiants discutent la structure cristalline observée et la confrontent avec la structure en nid d'abeille attendue; pour les aider dans l'interprétation de leurs résultats, une publication scientifique de référence leur est fournie [11]. On aborde ainsi les notions de liaisons chimiques fortes (liaisons covalentes entre les atomes de carbone qui constituent la couche de graphite) versus faibles (liaison de Van der Waals entre les atomes de carbone dans la direction d'empilement des couches).

Ces différences de liaisons entre les atomes de carbone dans le plan et entre les plans expliquent pourquoi il est possible d'exfolier ce matériaux, méthode basique et originelle qui a permis d'obtenir les premières monocouches de graphite, aujourd'hui appelé graphène. Le graphène présente des propriétés électriques, mécaniques, thermiques remarquables qui en font un nanomatériaux aux applications potentielles très variées [12] et il fait l'objet d'intense recherches financées au niveau européen par le programme «flagship graphène» [13].

Une fois les images atomiques obtenues, les étudiants réfléchissent aux différences auxquelles s'attendre si à la place du graphite ils sondaient par STM un échantillon de Graphène ; l'article de référence [11], leur fournie des pistes de réflexion.

Enfin ils étudient la dépendance du courant tunnel avec la distance pointe-surface puis avec la tension pointe-surface. A partir des courbes courant-tension pour une distance pointe-surface fixe, ils identifient deux régimes distincts de dépendance du courant tunnel avec la tension [14]: le régime tunnel classique et celui assistée par champ électrique (régime dit d'émission de champ ou de Fowler-Nordheim). Ce dernier présente un intérêt applicatif fort car il joue un rôle clés dans le fonctionnement des mémoires Flash [15] qui sont une alternative au disque dur classique pour stocker l'information dans les appareils numériques. 

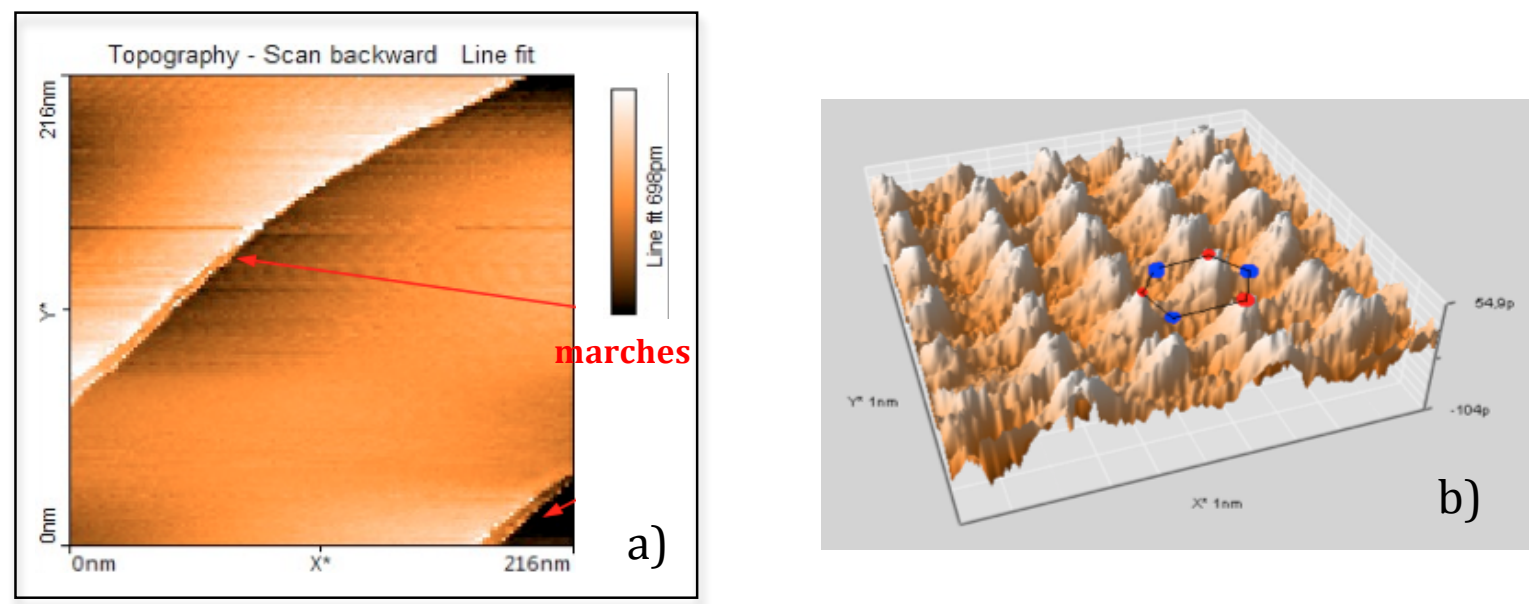

Figure 4: images d'une surface de graphite HOPG obtenues en TP par les étudiants; a) image à grand champ de $216 \mathrm{~nm}$ de côté où l'on distingue deux terrasses plates à l'échelle atomique séparées par des marches atomiques; b) vue en 3D d'un image d'une zone de 1nm de côté obtenue sur une terrasse, l'hexagone représente la maille du réseau cristallin du HOPG. On observe clairement que le STM ne permet de distinguer qu'un atome sur deux, ceci est lié à l'empillement des couches dans l'axe cristallin $\widetilde{C}$ dans lequel un atome de carbone sur deux a son voisin deux fois plus éloigné.

\subsection{Etudes des forces nanoscopiques et Nanomanipulation grâce aux "serious games"}

La présence de forces à l'échelle nanoscopique ainsi que leur origine et portée spatiale n'est pas une notion facile à mettre en évidence expérimentalement au cours d'une séance de TP de $4 \mathrm{~h}$ avec des appareils de type AFM. C'est pour répondre à ce besoin crucial de compréhension des forces régissant le nanomonde que le démonstrateur 'NanoLearner' (figure 5) a été développé[8]. Ce démonstrateur NanoLearner† combine la réalité virtuelle et un système à retour d'effort ou encore appelé système haptique. Ainsi une scène virtuelle a été conçue pour illustrer le Nanomonde et couplée à un système haptique qui permet de piloter un nano-objet virtuel, dans le cas présent une sonde AFM modélisée par un système masse-ressort, et de ressentir les effets des actions menées dans la nanoscène virtuelle [9].

A travers l'interface logicielle, les étudiants varient trois principaux paramètres de la force pointe-surface: la tension électrique (terme électrostatique qui illustre les forces à longues portées attractives, le rayon de la sonde AFM qui a un impact fort sur l'intensité de la force de Van der Waals et enfin le terme répulsif).

Dans la seconde partie du TP les étudiants expérimentent les stratégies de manipulation d'un nano-objet sur une surface (figure 5). Plusieurs paramètres sont alors variés tels que la rigidité de la surface, le type d'interaction entre l'objet et la surface et/ou la pointe AFM... Au cours de leur exploration, les étudiants prennent conscience que les stratégies de manipulation à l'échelle nanoscopiques différent fortement de l'échelle macroscopique. On discute l'origine des ces différences et cela permet de souligner le faible impact de la force gravitationnelle devant les autres forces (adhésion et friction) sur les objets nanométriques.

Dans leur CR, les étudiants doivent réfléchir sur le concept de "sérious game" et proposer un scénario que l'on pourrait implémenter sur le NanoLearner pour aider à la compréhension d'autres phénomènes physiques ou chimiques. Une de leur réponse favorite est : l'étude des différentes liaisons chimiques et la construction de molécules.

\footnotetext{
† Florence Marchi a reçu le le prix CETSIS 2010 d'innovation pédagogique pour ce démonstrateur
} 


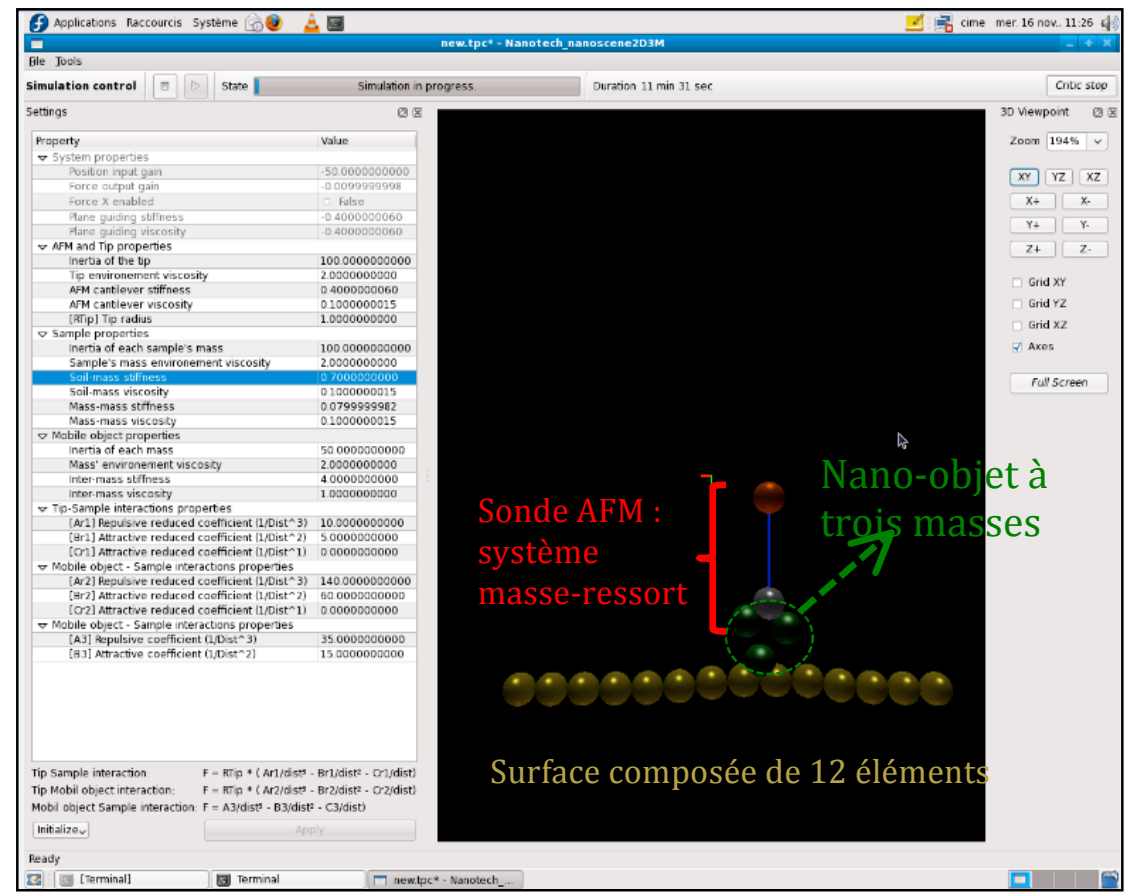

Figure 5 : Image du

logiciel du « serious game » le NanoLearner dédié à l'exploration du Nanomonde par une sonde AFM pilotée par un système à retour d'effort [8].

Inset: système haptique qui pilote la sonde AFM virtuelle et permet de ressentir les interaction sonde-échantillon

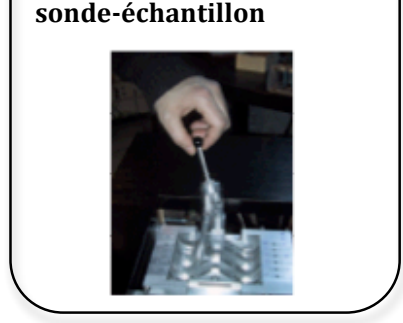

\subsection{Cellule photovoltaïque: les nanoparticules au service de l'énergie}

L'objectif du TP est de monter et de caractériser une cellule dite de "Grätzel » à base de colorant[16]. La réalisation de cette cellule passe entre autre par l'élaboration d'une suspension de nanoparticules de $\mathrm{TiO}_{2}$ obtenue par broyage/mélange mécanique de poudre de $\mathrm{TiO}_{2}$ et d'une solution d'acide acétique et une goutte de liquide vaisselle. La cuisson du film à haute température permet d'obtenir un film poreux homogène et résistant à l'ajout des électrolytes nécessaires à la bonne marche de la cellule.

Une fois la cellule montée (figure 6), les étudiants caractérisent son fonctionnement ainsi que son rendement par des mesures de courant et de potentiel à l'aide d'un potentiostat en plaçant la cellule devant une source lumineuse (une lampe de rétroprojecteur).

Ce TP est l'occasion pour les étudiants de mettre en pratique dans un cas très concret leurs connaissances de base en électrochimie, en électricité et en photochimie (absorbance des différentes molécules). Ils expérimentent la possibilité de réaliser un dispositif utilisant des nano-objets à bas coût dans un environnement peu contrôlé et les limites de reproductibilité que cela induit. De plus, ils se confrontent à un cas concret d'utilisation de nanoparticules dans le domaine porteur de l'énergie. Les avantages et les inconvénients de cette cellule photovoltaïque à colorant sont finalement comparés à ceux d'une cellule photovoltaïque à base de silicium.

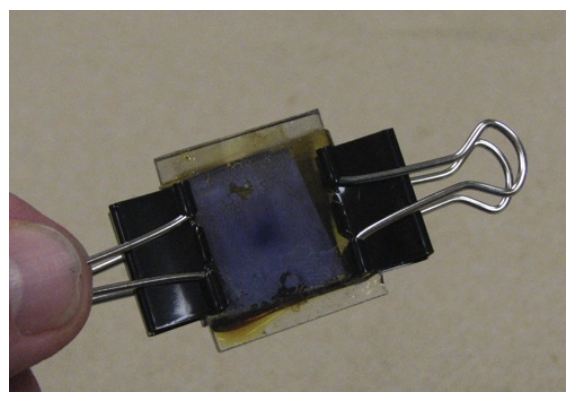

Figure 6 : Exemple de cellule réalisée par les étudiants 


\subsection{Caractérisation multi-échelle d'un polymère d'origine naturelle l'amidon.}

Les polymères sont des systèmes de taille nanométrique capables de s'auto-organiser jusqu'à l'obtention d'objets de formes et tailles variées. L'étude de tels objets oblige très souvent à les étudier à différentes échelles en utilisant plusieurs techniques de microscopie (figure 7). S'il est commun de retrouver aujourd'hui dans une salle de TP une binoculaire ou un microscope optique, peu de personnes ont accès à un microscope électronique à transmission ou à balayage. Les étudiants ont l'occasion de pouvoir appréhender le fonctionnement de ces différents appareils avec un technicien spécialisé dans ces différentes techniques.

Leur étude consiste à regarder des grains d'amidon de différentes origines biologiques (riz, mais, pomme de terre) en combinant microscopie optique et électronique. Tout d'abord sous un microscope optique en lumière polarisée ils peuvent mettre en évidence la biréfringence des cristaux (lien avec leurs cours d'optique) et leurs différentes formes suivant leur origine, puis ils métallisent un échantillon pour l'observer au MEB. Ils peuvent ainsi constater les différences entre une étude optique liée à la structure interne de l'échantillon et le MEB liée à la surface de celui-ci.

Enfin ils assistent à la réalisation d'une coupe mince d'un grain d'amidon à l'aide d'un ultramicrotome et son étude au MET (Microscopie Electronique à Transmission) par le technicien de la plateforme. Les étudiants peuvent ainsi se rendre compte des effets d'endommagement d'un échantillon sous le faisceau électronique à fort grossissement et les difficultés d'obtenir un bon contraste avec cette technique de microscopie (besoin de faire une «coloration » par un sel métallique lourd par exemple l'acétate d'uranyle)[17]. Cependant ils constatent le changement d'échelle qui est réalisé lors du passage du MEB au MET dont la résolution est de l'ordre du nanomètre.

D'un point de vue instrumental, il est aussi possible de noter la grande différence de fonctionnement entre un MET (image 2D) et un AFM (image 3D) en terme de résolution et de vitesse d'acquisition (balayage rapide en MEB ou MET contre plusieurs minutes pour un AFM) mais aussi leur complémentarité.
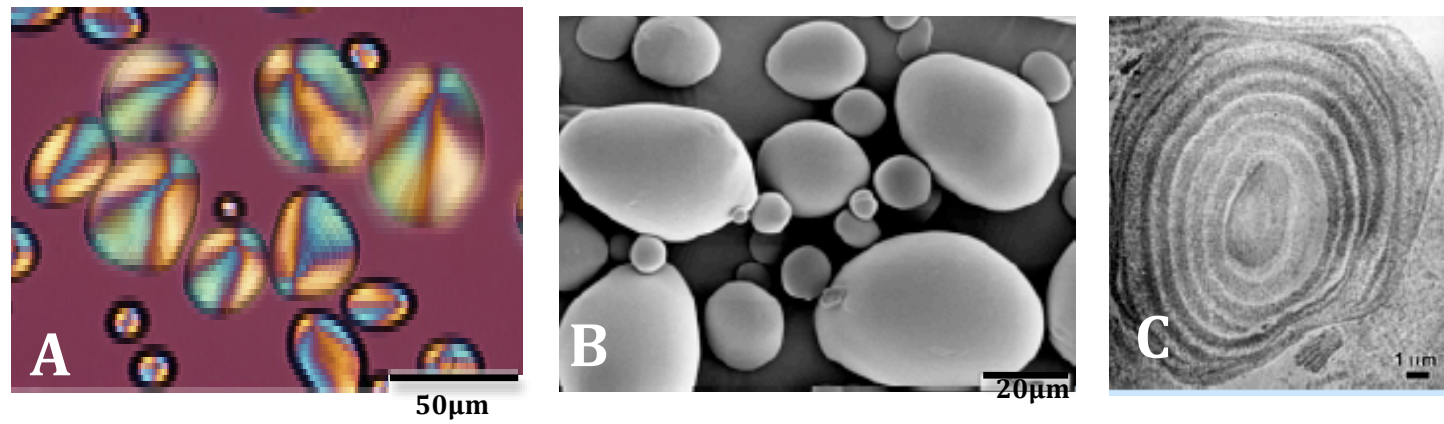

Figure 7: Exemples d'images d'un grain d'amidon réalisées par les étudiants en TP, A) en microscopie optique, B) en MEB, C) MET.

\section{Le contrôle des connaissances : conception et présentation d'une séance pédagogique}

Nous avons souhaité que l'évaluation de cette UE d'ouverture diffère de l'évaluation classique des UE destinés principalement à la préparation au concours. Nous voulions lui conférer un caractère innovant se rapprochant du travail de préparation qu'un enseignant doit mener pour traiter d'une thématique s'écartant du programme. En effet, pour ce style d'activité, l'enseignant ne peut pas s'appuyer simplement sur des livres de lycée, il doit alors se documenter pour s'approprier le sujet, construire ses propres documents, exercices et activités expérimentales. C'est dans cet esprit que nous proposons aux futurs professeurs de choisir un sujet "Nano" dans une liste que nous leur soumettons. Pour chaque sujet, les enseignants mettent à disposition un document de référence à savoir des articles en anglais tirés de revues de recherche prestigieuses comme «Nature» ou «Science» sur lequel les étudiants peuvent s'appuyer comme base de 
départ. Ils sont bien sûr encouragés à élargir leur recherche de documents et supports numériques (vidéo, animation, etc.). Le rôle de cet article de référence est de leur fournir un apport scientifique solide et valide sur le sujet traité, ce qui doit les aider par la suite à juger de la pertinence des autres sources qu'ils trouveront notamment sur internet.

Les sujets proposés aux étudiants adressent aussi bien des thématiques de nanophysique et nanoélectronique que de nanochimie en passant par la nanomédecine comme le montre la liste de 2011:

-Le carbone dans tous ses états

-Les nano-résonateurs

- Nanomédecine: diagnostique, imagerie
thérapie

Une fois un sujet choisi par un binôme d'étudiants, ils ont plusieurs semaines pour concevoir une séquence pédagogique qui s'articule autour d'un article de vulgarisation, d'un questionnaire à choix multiple (QCM), d'un exercice et enfin d'une activité complémentaire.

Chaque binôme doit rédiger un article de vulgarisation de deux pages (figures incluses) destiné à des élèves de niveau première ou terminale scientifique. Cet article doit s'accompagner de la conception d'un QCM contenant 4 à 6 questions, dédié à évaluer la compréhension des lycéens qui liraient leur article de vulgarisation. Pour compléter cette évaluation, le binôme doit concevoir un exercice sur le thème Nano choisi qui fait appel à des compétences de physique et/ou de (bio)chimie liées au programme du lycée. Ce travail demande aux étudiants de recontextualiser des connaissances et savoir-faire du programme de lycée à une thématique émergeante. Enfin le binôme doit proposer une activité complémentaire qui peut être de nature variée (activité expérimentale, visite d'entreprise, de laboratoire ou de CCSTI-Centre de Culture Scientifique Technique et Industrielle- rencontre avec un chercheur) afin d'illustrer de manière concrète les applications actuelles ou potentielles liées à ces thématiques.

L'ensemble de ces documents constitue un dossier pédagogique que chaque binôme remet au jury sous forme électronique (fichier PDF) une semaine avant la présentation orale d'une vingtaine de minutes qui comprend deux parties.

La première (10mins) est dédiée à exposer les grandes lignes du sujet choisi ainsi que les principaux aspects physiques et chimiques s'y rapportant; le niveau scientifique de cet exposé est le Master. Cette première partie est destinée à évaluer le degré de compréhension et la maîtrise de la thématique Nano choisi par le binôme.

La deuxième partie $(10 \mathrm{mins})$ s'attache à décrire la séquence pédagogique conçue à destination des lycéens et sa mise en pratique.

Cette présentation orale est suivie de 10 minutes de questions et discussion avec le jury portant sur des questions de compréhension du sujet scientifique ainsi que sur la démarche pédagogique proposée.

\section{Retour d'expérience et Perspectives}

Tous les binômes ont fourni des dossiers dont la qualité a été évaluée de moyenne à excellente, tous ont rendu un dossier complet comprenant l'ensemble des trois parties requises. La relative liberté de forme pour élaborer l'exercice associé a permis à certains étudiants de réinvestir des méthodes pédagogiques apprises dans d'autres UE; par exemple l'élaboration d'un exercice proposé avec différents niveaux de difficultés parmi lesquels les élèves devront choisir en fonction de leur capacités. Ce dispositif pédagogique découle directement de l'apprentissage par «tâche complexe»[18] auquel les étudiants ont été formés dans une UE de didactique des sciences.

Afin d'améliorer le fond et la forme de cette UE non-conventionnelle au sein du master enseignement, nous récoltons l'avis des étudiants sous forme de questionnaire. De manière générale, les étudiants trouvent cette UE intéressante à la fois pour ses nombreux TP, son contenu et pour sa forme d'évaluation. Ils soulignent l'intérêt fort des TP qui illustrent de manière concrète des notions qui leur paraissent souvent abstraites (effet tunnel par exemple) 
et des appareils qui leur sont peu connus (MEB/TEM et microscopie en champ proche).

Cependant ils sont souvent surpris par le temps important nécessaire à la préparation de ce dossier pédagogique et de l'exposé associé.

Ils apprécient la mixité du jury où se mêlent enseignants du supérieur et du secondaire car cela leur permet d'être confronté à des questions variées portant sur la compréhension du sujet choisi, des questions matérielles de mise en place dans la classe de l'activité expérimentale en passant par des discussions sur l'approche pédagogique.

Dans la partie du questionnaire relative aux suggestions d'amélioration, des propositions intéressantes émergent, comme le désir de pouvoir suivre une journée de «Nano@school»qui a lieu au CIME-Nanotech. Cette demande ainsi que la pertinence de la mise en place de cette UE "Objets Quotidiens, Nanosciences et Education» prend une toute nouvelle dimension depuis l'introduction officielle de thématiques «Nano» dans les programmes de PC au lycée en 2011 (première S) et à la rentrée 2012 (Terminale S).

On peut s'interroger sur l'absence au cours de cette UE, de sensibilisation des étudiants aux questions sociétales et éthiques que soulèvent les nanosciences et nanotechnologies. En effet l'impact toujours croissant des sciences et techniques dans nos modes de vies, implique de former les citoyens pour qu'ils puissent s'approprier ces sujets afin de pouvoir participer de manière active et éclairée aux choix de société qui en découlent. Une des voix privilégiées pour initier les futurs citoyens aux controverses scientifiques et sociétales, est d'organiser des débats en classe animés par les professeurs. Cependant, il a été montré par de précédentes études[19,20] que les professeurs de science y sont souvent réticents. Les principales raisons de ces réticences sont liées d'une part à leur manque de maîtrise scientifique et technique du sujet et d'autre part de méthodologie dans l'organisation et le déroulement du débat. Nous avons donc choisi de focaliser l'UE «Objets quotidiens, Nanosciences et Education» sur l'acquisition et la restitution des connaissances sous un format pédagogique afin que les futurs enseignants sachent comment s'y prendre pour s'approprier une thématique scientifique "chaude». Cette étape nous semble être le prérequis indispensable pour organiser et mener un débat sur un sujet scientifique émergeant. Les méthodes pour organiser un débat autour de questions sociales et éthiques concernant des thèmes scientifiques «chauds " doivent faire l'objet d'une UE ou partie d'UE traitées par les sciences de l'éducation. Il serait donc très intéressant que les enseignants de sciences de l'éducation s'appuient sur les connaissances scientifiques et techniques acquises à travers l'UE «Objets quotidiens, Nanosciences et Education» pour illustrer leur enseignement sur une thématique scientifique émergeante sur laquelle les étudiants ont acquis une base en physique, chimie et instrumentation suffisante. Ce type d'activité pourrait être l'occasion de renforcer les liens entre les enseignants-chercheurs en sciences de l'éducation et ceux en nanosciences et nanotechnologies qui sont encore trop souvent distants dans le milieu académique en France.

\section{REMERCIEMENTS}

Tous nos remerciements pour leur participation au jury de l'UE «Objets quotidiens, Nanosciences et Education», à Evelyne Excoffon inspecteur d'académie-inspecteur pédagogique régional de sciences physiques de l'académie de Grenoble, à Bruno Pin et Eric Martinet tous deux professeurs de physique-chimie respectivement au lycée des Eaux Claires et au lycée international de Grenoble et pionniers dans l'implication de leur classe dans le programme Nano@school dès 2010.

\section{REFERENCES}

[1] http://phitem.ujf-grenoble.fr/formations-et-metiers/preparations-aux-concours-de-l-enseignement/

[2] http://www.cime.inpg.fr/

[3] http://physique-eea.ujf-grenoble.fr/MasterNano/

[4] http://pca.ujf-grenoble.fr

[5] http://www.nanoatschool.org/

[6] http://www.nano-ecole.fr/ 
[7] 'La plateforme NanoMonde: de la physique des interactions à l'échelle nanométrique à la nanocaractérisation des surfaces et dispositifs', F. Marchi, J. Chevrier, L. Montes, E. Pernot, S. Cornu, A. Luciani, Ch. Schaeffer, proceedings pp 175-181, 9ème journées Pédagogiques du CNFM (2006)

[8] «Le concept du NanoLearner : Les mains dans le Nanomonde de l'Université vers le grand public», F. Marchi, J. Castet, S. Marlière, N. Castagné, J. Chevrier, A. Luciani et JL. Florens, revue électronique J3eA Volume 9 (2010). http://dx.doi.org/10.1051/j3ea/2010017

[9] "An augmented reality nanomanipulator for learning nanophysics : the "Nanolearner" platform ", F. Marchi, S. Marlère, J.L. Florens, A. Luciani and J. Chevrier, LNCS 6250, Journal of Transactions on Edutainment IV, pp157-175, Springer (2010)

[10] “Silicon processing for VLSI Era”, S Wolf, Lattice Press, volume 4 - Deep submicron process technology- (2002).

[11] «Revealing the hidden atom in graphite by low-temperature atomic force microscopy», S. Hembacher, F.J. Giessibl, J. Mannhart, and C. F. Quate, PNAS, vol. 100 (22) ,pp 12539-12542 (2003)

[12] "Le graphène sort des labo", l'usine nouvelle, N 187393 (2012)

[13] http://www.graphene-flagship.eu/GF/index.php

[14] "Local spectroscopy and atomic imaging of tunnelling current, forces and dissipation on graphite", S. Hembacher and al, Phys. Rev. Lett. 94, 056101 (2005).

[15] http://www.presence-pc.com/tests/ssd-flash-disques-22675/2/

[16]"A Low-Cost, High-Efficiency Solar-Cell Based on Dye-Sensitized Colloidal $\mathrm{TiO}_{2}$ Films", B. O'Regan and M. Grätzel Nature 353, 737 - 740 (1991)

[17] "Problems Associated with the Electron Microscopy of Polymers" in "Electron Microscopy of polymer", chap8, G.H. Michler, Springer (2008).

[18] http://eduscol.education.fr/cid51827/temoignage-mise-en-oeuvre-dans-la-classe.html

[19] « Des enseignements de sciences dans une perspectives d'éducation citoyenne ? », Didaskalia, 33, 121-140, (2008).

[20] « Nanotechnology and nanoscale science : educational challenges», M.G. Jones, R. Blonder, G.E. Gardner, V. Albe, M. Falvo and J. Chevrier, International journal of science education (2013), DOI:10.1080/09500693.2013.771828 